\title{
Images et textes dans les mémoires professionnels du second degré
}

Isabelle de Peretti

\section{(2) OpenEdition}

1 Journals

Édition électronique

URL : http://journals.openedition.org/trema/3312

DOI : 10.4000/trema.3312

ISSN : 2107-0997

Éditeur

Faculté d'Éducation de l'université de Montpellier

\section{Édition imprimée}

Date de publication : 1 mai 2015

Pagination : $52-67$

ISSN : 1167-315X

\section{Référence électronique}

Isabelle de Peretti, «Images et textes dans les mémoires professionnels du second degré », Tréma [En ligne], 43 | 2015, mis en ligne le 25 juin 2015, consulté le 01 mai 2019. URL : http:// journals.openedition.org/trema/3312 ; DOI : 10.4000/trema.3312

Ce document a été généré automatiquement le 1 mai 2019.

Trema 


\title{
Images et textes dans les mémoires professionnels du second degré
}

\author{
Isabelle de Peretti
}

\section{Introduction}

1 L'image séduit, le texte ennuie. Tel est le postulat qui semble largement partagé, dans nos masters des métiers de l'enseignement, par les étudiants de Lettres Modernes, soucieux pourtant d'amener leurs élèves à la lecture, de partager avec eux leur amour de la littérature, de développer leur goût de l'écriture tout en donnant sens à leurs apprentissages. Ainsi les recours à l'image ou aux arts visuels en général, et surtout à la bande dessinée, à la photo, au tableau, au film, plus rarement au théatre, tendent à prendre de plus en plus de place dans leurs projets, leurs séquences ainsi que dans les problématiques de leurs mémoires professionnels. Ce postulat, ces choix, ne sont certes pas nouveaux (cf. Veck, 1994; Beaudrap (de), 2004); toutefois le contexte culturel, marqué par l'avènement du numérique et le contexte institutionnel ne peuvent manquer d'infléchir significativement la réflexion et les pratiques de ces enseignants en formation : trois nouvelles prescriptions ${ }^{1}$ et une nouvelle épreuve pour la certification de fin de scolarité obligatoire ${ }^{2}$ viennent, en effet, de modifier les orientations du travail des enseignants dans ce sens.

2 Le recours à l'image sous toutes ses formes dont s'emparent avec facilité nos étudiants a$\mathrm{t}$-il donc une simple fonction de motivation, d'enrôlement dans les travaux disciplinaires ou s'agit-il de la construction d'une culture humaniste ${ }^{3}$ qui articulerait dans la conscience claire de la spécificité de leurs objets et de leurs méthodes, les disciplines scolaires du français, de l'histoire et des arts visuels, voire qui permettrait plus de clarté épistémologique sur ces disciplines (transdisciplinarité ou épistémologie comparée ${ }^{4}$ ) ? Ou ce détour par l'image, s'il s'agit d'un détour, n'est-il pas porteur d'un risque d'esquive des difficultés propres à chaque discipline, d'un escamotage en définitive préjudiciable aux élèves (Bautier et Rochex, 1998), le risque étant alors « de revenir des disciplines avant 
d'y être allé [...] », et, « En cherchant à tisser des liens entre tout ...et tout, (...) d'aboutir à une pensée ouverte à tous les vents!» (Astolfi, 2008: 151). Et, dans la discipline du français, d'escamoter les apprentissages fondamentaux en terme de lecture et d'écriture, notamment.

Qu'en est-il donc, dans les pratiques de ces jeunes enseignants et dans l'état actuel de la formation? C'est donc cette problématique que nous nous proposons d'aborder ici, en étudiant, dans une visée essentiellement descriptive, un corpus de mémoires professionnels d'étudiants du master MEF en Lettres Modernes. Après avoir précisé les cadres et la méthodologie de ce travail, nous aborderons les référents explicites ou implicites présents dans les mémoires pour cerner leurs objets, les éléments du programme et les œuvres choisies dans ce recours à l'image, puis les approches didactiques privilégiées dans ces manières d'enseigner, en lien avec les questions d'épistémologie scolaire qu'elles soulèvent, dans le cadre limité de ce petit corpus.

\section{Contexte de la réflexion, cadre de référence et méthodologie}

\section{1. Matrice du français et instructions officielles}

4 La matrice disciplinaire du français se dégageant notamment des instructions depuis celles de 1996 pour le collège peut être référée à la définition donnée par Y. Reuter (1995 : 244) comme " production/réception des discours oraux et écrits)». Parmi ceux-ci, bien sûr et au centre des travaux des étudiants, le discours littéraire, spécifié par le concept ou la notion de lecture littéraire avec les différentes conceptions qui la soustendent (Dufays, 2005), par les conceptions de l'activité du sujet-lecteur (Rouxel et Langlade, 2004), par des approches génériques, stylistiques des textes, mais aussi par des conceptions scolaires de l'écriture ou de l'apprentissage de la langue. Dans les instructions de 1996, l'interdisciplinarité entre le français et les enseignements d'arts plastiques (lien image-texte), d'histoire et d'éducation est déjà explicite (p. 16).

Dans les instructions officielles du 28 août $2008^{5}$ pour le collège, l'enseignant de français voit, une fois de plus, le champ de ses missions s'agrandir, il doit dispenser les éléments d'une culture qui permette «la compréhension des œuvres littéraires, cinématographiques, musicales et plastiques", qui "contribuent également à la fondation d'une culture humaniste ». Cette ouverture aux autres arts parait également être au service de la compréhension des œuvres littéraires étudiées, en éclairant les contextes historiques et culturels (dans une démarche d'interdisciplinarité) mais aussi au service de la meilleure appréhension des méthodes d'analyse littéraire, dans une démarche plus transdisciplinaire, mais dont la littérature reste le centre. En effet, on y lit "L'image, quant à elle, favorise l'expression des émotions et du jugement personnel ; elle peut en outre consolider l'apprentissage des méthodes d'analyse ». Par ailleurs, il est également spécifié que l'approche de l'image se fait selon des démarches proches de celles de la lecture des textes, ce qui peut tendre à gommer les spécificités et les apports mutuels des diverses disciplines artistiques. L'image doit être toujours « en relation avec des pratiques de lecture, d'écriture ou d'oral ", en préparation, accompagnement, prolongement des textes. Elle doit faire le lien avec l'histoire des arts, ce qui, de fait, dans ce texte, met au premier plan les arts visuels dans cette démarche principalement interdisciplinaire. 
6 L'approche préconisée dans le Socle commun des connaissances $(2006)^{6}$ est conçue dans le cadre européen de la constitution d'une culture commune à l'Europe, dans une perspective humaniste que l'on peut référer à Erasme (Musset, 2008). L'accent est mis sur une définition anthropologique de la culture, accordant une place centrale à la perspective historique, aux textes patrimoniaux, favorisant la rencontre avec les œuvres littéraires et artistiques « majeures » « du patrimoine français, européen et mondial » (p. 8), dans une tension entre ouverture à la diversité des cultures et universalité des droits de l'homme. La transdisciplinarité y est favorisée (formation du jugement, du goût, de la sensibilité), « chaque compétence [...] requiert les contributions de plusieurs disciplines » (p.2): pour le pilier 5 de «la culture humaniste», les champs disciplinaires convoqués sont ceux de l'histoire, de la géographie, des disciplinaires littéraires et artistiques.

7 L'enseignement de l'histoire des arts ${ }^{7}$, également, participe de la constitution de cette culture commune dans sa dimension historique et dans une perspective curriculaire de l'école au lycée. Il s'agit d'un enseignement transversal, tous les enseignants et potentiellement toutes les disciplines (pas seulement celles du domaine " humaniste ») étant concernés. Certains des objets de la discipline du français se retrouvent dans deux des six grands domaines artistiques qui constituent l'un des trois piliers de ces instructions, à savoir dans les « arts du langage » ou dans les « arts du spectacle vivant». Il s'agit toujours de rencontres « sensibles et réfléchies » avec l'œuvre d'art, le lien entre la connaissance et la sensibilité étant visé, les disciplines scientifiques n'étant pas exclues. Le double dialogue des différentes disciplines entre elles et avec les disciplines artistiques et des disciplines artistiques avec les autres disciplines y est encouragé. Les formes et genres visés sont plus explicitement ouverts à un corpus contemporain et à des manifestations artistiques plus diverses, le terme d'œuvres "majeures» disparait d'ailleurs au profit de celui d'œuvres «significatives». "Ces domaines artistiques sont explorés dans leurs manifestations patrimoniales et contemporaines, populaires et savantes, nationales et internationales, occidentales et non occidentales» (p. 3). En lien avec des partenariats culturels, l'organisation préconisée situe l'histoire des arts dans une visée de rénovation pédagogique, de constitution d'une communauté éducative, car elle « instaure des situations pédagogiques pluridisciplinaires et partenariales » (p.1). S'agit- il alors plutôt de pluridisciplinarité, et des pratiques interdisciplinaires, voire transdisciplinaires y ont-elles leur place (cette prescription manifestant une certaine instabilité lexicale sur ce plan)? Dans ce contexte institutionnel, comment penser des pratiques au service des disciplines et de leurs articulations, de leurs apports réciproques, ou de véritables constructions transdisciplinaires?

\section{2. Corpus des mémoires professionnels et méthodologie}

8 Les mémoires professionnels ont été préparés et encadrés en seconde année de master à la fois dans un séminaire de didactique du français et dans les enseignements de culture disciplinaire professionnelle (ou mise en situation professionnelle) préparant et analysant les stages (environ 54 heures en stage "filé ", un jour par semaine). Ils ont été évalués selon des critères spécifiques pour ces deux enseignements; plus qu'une formation à la recherche, il s'agit d'une formation par la recherche et par l'appui sur des travaux de recherche, l'activité du chercheur en didactique de français conçue en quelque sorte comme " pratique sociale de référence " (Martinand, 1986) étant toutefois valorisée dans les mémoires. Ces travaux ont été réalisés par les étudiants ayant suivi l'ensemble du 
cursus de master 1 et master 2 en 2010/2011 et 2011/2012 (à l'exception d'une étudiante arrivant de Tahiti), le concours du CAPES intervenant au cours de la deuxième année. Outre les enseignements littéraires, d'épistémologie et de didactique du français, les étudiants ont aussi bénéficié dans ce cursus en première année d'une courte formation à l'étude de l'image ( $15 \mathrm{~h}$ ) et de sémiotique du cinéma (15h) (sur un total de 570h en M1 et de $300 \mathrm{~h}$ en M2). Il s'est plus agi de juxtaposition que de relations organiques entre les différents enseignements ${ }^{8}$.

Les 18 mémoires produits ont été analysés à partir d'une grille comportant des éléments de description et de contextualisation des séquences ou séances analysées (problématisation, disciplines ou objets artistiques convoqués, sujets, références explicites ou implicites aux instructions officielles ou à d'autres influences, justification des choix opérés, impact perçu sur les élèves ), des éléments référés à la conception de la matrice disciplinaire de Develay (1995: 27) (définition des objets de savoirs, nature des problèmes posés et questions clés, références explicite ou implicite à des savoirs savants et/ou à des pratiques sociales de référence ou des savoirs d'experts, finalités et valeurs, méthodes d'approche de l'objet, éléments noyaux ou notions-clés dans les savoirs visés, tâches prescrites et genres scolaires, épreuves certificatives) et des éléments liés au type de relation entre les approches disciplinaires convoquées (pluri, inter ou transdiciplinarité) en référence à Astolfi (2008). Pour analyser le choix des pratiques exposées par les étudiants dans leurs mémoires, on se référera également à la typologie des discours de référence proposée par J.-C. Chabanne, M. Parayre, E. Villagordo (2011 : 79) : discours du sémiologue, du critique ou de l'amateur d'art, de l'historien de l'art, du philosophe de l'art, de l'herméneute, du poéticien et même de l'artiste lui-même. «[...] C'est bien à partir de ces discours de référence que les enseignants vont se repérer pour intervenir : encourager les conduites verbales qui s'orientent vers ces discours modèles, les reformuler dans ce sens, évaluer la pertinence des interventions, juger des compétences déjà en place et les valider etc. De même, la connaissance de ce que peuvent faire des experts est aussi une mesure de la difficulté réelle des tâches proposées, et permet d'évaluer positivement ce qui peut apparaitre, à première vue, comme des paroles malhabiles, dispersées, obscures, ce que Frédéric François désigne comme le difficile-àdire : « Une parole heurtée, maladroite de dire ce qui peut être ressentie comme la bonne façon de dire le difficile-à-dire «(François, 1999, p.29) » p. 91.

Enfin l'analyse de notre corpus, nous amène à envisager la catégorie de discours incitatif, préconisée par Dumortier $(2011,33)$, catégorie qui nous est apparue très favorable à la prise en compte des émotions esthétiques des élèves dans les mémoires étudiés. Notre position est ici celle du chercheur impliqué (partiellement) puisque nous y avons dispensé des enseignements (épistémologie et didactique du français, recherche et suivi de douze de ces mémoires). Bien entendu, nous travaillons sur des pratiques déclarées, sur du discours et non sur l'observation directe de situations de classe.

11 Trois mémoires ${ }^{9}(1,16,18)$ traitent de questions transversales à l'enseignement du français sans référence à d'autres disciplines de la culture humaniste : ils abordent «Les préparations de cours", "La consigne", "Les interactions entre élèves en cours de français ». Deux autres $(3,9)$, traitant respectivement de l'obstacle de la langue pour l'approche de la littérature et de l'enseignement de l'argumentation, n'envisagent pas non plus d'autre champ disciplinaire excepté et de façon brève sous la forme de l'analyse sémiologique de premières de couverture (pour Candide, L'Avare etc.). 
12 L'analyse fine portera sur les treize autres mémoires. Si quatre d'entre eux portent précisément sur l'histoire des arts et l'image ${ }^{10}$, les neuf autres, quel que soit leur sujet, intègrent l'analyse de bandes dessinées, de films, de tableaux, de photos ${ }^{11}$, les «arts de l'espace », « du quotidien », du « son $»^{12}$ n'étant pratiquement pas abordés.

\section{L'image : référents théoriques sémiologiques et approche didactique fonctionnelle}

\section{1. Définitions explicites de l'image}

13 On ne trouve qu'exceptionnellement de renvois explicites à un savoir savant de référence pour la définition de l'image ; quant il intervient, traitant de l'image en général, il se situe principalement dans une perspective sémiologique, avec des références à Barthes ( Mythologies, 1957) pour l'immédiateté du sens de l'image, qui « devient une écriture dès lors qu'elle est significative » (2), à ses articles dans la revue Communications (1964, 1975), à ceux de Metz (1970) (4), ou encore à des articles de Communication et langages 4 (Garnier, 1969), 20 (Escarpit, 1973), 24 (Almasy, 1974), 33 (Delannoy, 1982) etc. Au niveau de la discipline scolaire, on la trouve encore surtout spécifiée par les opérations mentales qu'elle permet (pensée divergente), caractéristique, selon Ardouin (1995, p.46), du paradigme des années 68-80 : le paradigme de la créativité.

\section{2. Référents implicites}

C'est donc surtout de façon implicite, en lien avec les références aux instructions officielles qui sont nombreuses, que l'image fixe ou mobile est définie et ce, en termes de réception, d'immédiateté sensible de sa réception. Dès lors, ce sont des effets qui sont pointés dans ce domaine :

- sensibles et esthétiques : favorisant l'expression des émotions, et l'émotion esthétique (13), ce qui « [...] peut produire une sorte de choc émotionnel (4) », " pas les mêmes canaux émotionnels que le discours abstrait qui s'intéresse davantage à l'intellect », « pouvoir de réaction plus important » $(4)$, sans les « barrières de la lecture » $(11$, p.7), « qui présente un sens à tout le monde (au-delà des différences linguistiques et culturelles)» $(4, \mathrm{p} .16)$, donc plus sensibles ici que véritablement esthétiques ;

- Cognitif (connaissances et compétences) : émergence des représentations ou apport rapide de connaissances (2) (6) (9), contextualisation des œuvres étudiées (9), sens de l'observation (6), attention, contribution à la formation de la culture, au développement de l'imaginaire (6), de la faculté d'interprétation (hypothèses, questionnement), également pensée divergente (4) ;

- motivationnel et pédagogique : motivation (tous les mémoires), effet de rupture par rapport aux routines scolaires (11), diversification par prise en compte. Elles facilitent également la création d'une communauté interprétative $(2, \mathrm{p} .8)$.

15 L'image est ainsi auxiliaire du texte ou support pour les évaluations diagnostique et finale, la structuration, la mémorisation, la récapitulation et le réinvestissement des connaissances. L'entrée par la place ou la fonction dans la séquence d'apprentissage est assez souvent attestée. Un seul mémoire insiste sur le fait que l'image n'est pas assez étudiée pour elle-même. 

stylistiques et textuels, par confrontation à ceux des textes (11) : «Selon Sylvie Anahory, "l'image aide les élèves à une meilleure compréhension des objets stylistiques et textuels ${ }^{13}$ » ou à la culture artistique qu'elle permet.

17 En dehors de ces éléments de réception, le recours à l'image est aussi justifié par des références à l'évolution culturelle et sociale (multiplication des images dans la société, « jusqu'à en donner le tournis » (4), évolution du rapport aux livres, à l'écrit des élèves, à la culture des élèves (10).

On observe par ailleurs dans les mémoires une instabilité sémantique et générique, on glisse souvent de l'image et de l'analyse de l'image, tous supports confondus (affiches, publicité, cinéma, vidéo, tableaux, sculpture etc.) aux productions artistiques plastiques, à l'histoire de l'art et à l'histoire des arts. En termes de discours ou de pratiques sociales de référence, on le verra, si le discours du sémiologue est présent dans les tâches proposées aux élèves, comme celui de l'historien de l'art, peu défini, est pourtant plus fréquent.

\section{3. Questionnements}

19 C'est évidemment l'approche motivationnelle qui domine, comme en témoigne d'ailleurs de nombreux titres de mémoires : «L'image, auxiliaire de la discipline du français ? (2); «L'image comme déclencheur de l'écriture » (10); «Motiver par l'histoire des arts" (11); et de façon plus implicite «La variété des supports» (6), ainsi que «Ce qui motive ou rebute l'élève à la lecture des pièces de théâtre "(9).

Une étudiante qui a consacré son mémoire à l'impact de l'histoire des arts sur la motivation des élèves au collège conclut ainsi :

«Le recours à l'histoire des arts paraît influer sur la motivation des élèves: ils semblent plus désireux de s'investir et de faire progresser le cours. Et ce qui est remarquable, c'est que cette motivation est durable : les élèves qui s'intéressent à l'histoire des arts montrent une participation plus ou moins égale lors de chaque séance. Néanmoins, il faut noter que cette motivation ne s'applique pas à tous. Dans chaque niveau observé, seuls deux à quatre élèves portent un intérêt véritable et profitable à cet enseignement. En outre, on peut ajouter que cet enseignement est davantage apprécié dans les classes des niveaux les plus élevés. » (11).

Mais l'évolution des problématiques exposées dans les mémoires montrent un glissement du questionnement vers le rôle de l'image dans les apprentissages, vers la pertinence des choix, la fonction des images dans les apprentissages littéraires et langagiers visés, leur impact réel sur les connaissances et les compétences: on va ainsi de l'approche de la littérature et de l'écriture par l'histoire des arts, à leur impact sur la meilleure compréhension de la littérature et une meilleure expression (11); de «Comment motiver par l'image? » à «En quoi l'image répond-elle à quatre types de fonctions : inductive, élargissement, validation, éducation?» (4); à "Comment l'image structure-t-elle les connaissances?» $(4$, p. 25$)$ ou «Comment et quand exploiter le support image? Quels sont les avantages et les inconvénients sur le plan pédagogique et didactique? $(4$, p.31). Cette orientation par les fonctions, présente dans les instructions officielles, se retrouve d'ailleurs dans plusieurs mémoires : deux d'entre eux se réfèrent explicitement au travail de Patricia Kalhart (2002) pour l'un d'eux, en référence centrale, pour l'autre en référence combinée avec une approche plus culturelle. 


\section{Culture de l'image et programmes de français : plutôt le patrimoine?}

Sans surprise, c'est à l'occasion de l'étude des textes fondateurs que, en accord avec l'orientation préconisée du Socle commun (2006), les arts visuels (les tableaux principalement, mais aussi les bandes dessinées), sont convoqués, soit dans cinq de treize mémoires, à l'occasion de l'étude de l'odyssée (épisode des sirènes, Circé) $(2,11)$, de la Bible (La Génèse) (12) ou des Métamorphoses d'Ovide $(8,17)$.

3 Sans prendre en compte les prolongements et recherches demandés sur ces sujets aux élèves, on notera que sont étudiés les œuvres et supports suivants (souvent d'ailleurs convoqués dans les mémoires sans mention de dates):

- des photos de représentations murales, de poteries (vase grec du Ve siècle) (2);

- des photos de sculptures : de l'antiquité (2), d'autres époques : Homère sculpté par PhilippeLaurent Roland (1812) (2), Enlèvement d'une sabine, de Jean de Bologne (1582) (11);

- des tableaux : Ulysse et les sirènes de L.-A. Adolphe Belly (1867), Ulysse et les sirènes de John William Waterhouse (1891), Ulysse et les sirènes de Herbert Draper (1909) (2), deux représentations de Circé de John W. Waterhouse (11), La reproduction de la fresque de la chapelle Sixtine : La création d'Adam de Michel Ange $(1510)(4,11)$, Adam et Eve $(1526)(4,11)$ ou Adam et Eve au Paradis de Luca Cranach l'Ancien (12), Les Sabines de Jacques-Louis David (1796-1799) (11) ;

- la bande-dessinée L'Odyssée de Christophe Lemoine et M. L. Imbiriba (2009, ed. Glenat) (2), et La bible, l'ancien testament en $\mathrm{BD}$, ouvrage donné sans référence (12) ;

- un corpus de couverture d'éditions scolaires récentes de L'Odyssée (11);

- des affiches publicitaires récentes (8), (11), (12);

- des extraits de films : de la saga de Twilight et du film Harry Potter et le prisonnier d'Azkaban, issu du livre de J. K. Rowling (1999) (8), Pirate des Caraibes, La fontaine de jouvence (Rob Marshall, 2011), de téléfilms Desperate Housewives... ;

- une photo de F. Luckasseck (2005), Cyprès sur une route de campagne, présente dans un manuel (17).

C'est aussi logiquement mais de façon un peu moins nette, à l'occasion de l'étude d'un mouvement littéraire et culturel, l'humanisme (13), le romantisme (4) ainsi que pour la poésie engagée (5) ou encore lors de l'approche de genres: la comédie au XVIIe (9), l'autobiographie $(6,10)$, ou d'œuvres patrimoniales : textes du Moyen Âge, Les Misérables d'Hugo, la poésie d'Arthur Rimbaud (6). On y retrouve les œuvres et documents suivants :

- des tableaux : La vierge aux rochers (1483/1490) et la Joconde (1503/1506) de Vinci, Les Ambassadeurs de Hans Holbein (1533), la Venus d'Urbino de Titien (1538/1539) et Les noces de Cana de Véronèse (1562) (13), Le radeau de la Méduse de Gericault (1818/1819) (4), La colonne brisée de Frida Kahlo (1944) (10), Guernica de Pablo Picasso (1937 ) (10), Autoportrait au christ jaune et Le Christ jaune (1889) de Gauguin (10) ;

- des illustrations d'œuvres littéraires par un artiste (pratiques hyperartistiques (Genette 2013) ou échanges intersémiotiques) : des Fables de La Fontaine par Félix Lorioux notamment « La cigale et la fourmi » (1926) (10), du poème Liberté d'Eluard par Fernand Leger (1953) (5), l'illustration du Dormeur du Val de Rimbaud par Ida Polo (6) ; 
- des bandes-dessinées : Les misérables de Victor Hugo (donnée sans références), L'enfant de Jules Vallés, par Laurence Harlé et Sylvain Bourrières (17), Ubu roi (Luc Duthil et A. Petit (2007), des extraits du manga Gen d'Hiroshima (1973-1985) de Keiji Nakasawa (6) ;

- des extraits de films : Le dictateur de C. Chaplin et V pour Vendetta d'Alan Moore, le film Perceval Le Gallois de Rohmer (17), Un secret de C. Miller (2007) tiré de l'autobiographie de Philippe Grimbert (2004), le dessin animé Kirikou et la sorcière de Michel Ocelot (1998) (12), Molière d'A. Mnouchkine (date) (9), un film sur la vie de Rimbaud Eclipse totale d'A. Holland, 1995 (6) ;

- du théâtre filmé, des extraits de la mise en scène de P. Fox des Fourberies de Scapin ;

- une étude d'affiche ;

- le chant des partisans et la version récente de Zebda (2007), des contes enregistrés par un artiste (12). pratiques d'experts, soit des livres d'artistes pris au sens large, poème-objet ou poème pictural par exemple pour les panneaux peints par Fernand Léger pour illustrer le poème Liberté d'Eluard à la mort du poète. Ces sous-genres qui peuvent, pour J.-C. Chabanne et J.-L. Dufays $(2011,14)$, servir d'objets d'études et de références pour penser la parole sur l'œuvre en classe. De fait, cette œuvre, a servi de modèle de référence dans une classe pour la confection, en lien avec l'écriture de poèmes engagés, d'affiches qui les mettront en valeur (cf. ci-dessous) (5).

Si la visée première de ces apports parait être de rendre attractive cette culture patrimoniale, d'en faciliter l'accès, comment se nouent précisément les relations entre l'enseignement du français et les disciplines artistiques dans les pratiques de classe?

\section{Autour de l'image, dans les classes}

On étudiera principalement ici les quatre mémoires qui ont choisi l'image comme objet central $(2,4,10,11)$, en tant que représentatifs des tendances du corpus et on complétera ces analyses par des données prises dans les mémoires $(13,10,6,5)$, abordant cette question de façon transversale. 


\section{V.1. Les difficultés} ce domaine, on examinera les impasses, les difficultés rencontrées, signalées par les étudiants. Les choix d'images peuvent bien sûr tout d'abord se révéler inutiles sur le plan des apprentissages : par exemple, une étudiante note que l'extrait du film sur la vie de Rimbaud Éclipse totale d'A. Holland, 1995, n'apporte rien pour l'étude du poème «Le Dormeur du Val » (6) ; Des tableaux ou des illustrations choisies trop rapidement peuvent se révéler contre-productives: c'est ce que constate une étudiante à propos d'une anticipation censée préparer l'étude du poème «Melancholia » de V. Hugo (10). Les manuels sont d'ailleurs examinés de façon critique de ce point de vue (images « gratuites » en ouverture des chapitres par exemple) (4). Les étudiants sont aussi parfois surpris de constater que les élèves n'adhérent pas tous sans réserve aux bandes dessinées, qui brident leur imagination. Enfin une étudiante constate l'amoindrissement du pouvoir des images qui sont de plus en plus fréquemment utilisées en classe, entrainant à leur tour des effets de routine (4).

Si la volonté de souscrire à cette éducation esthétique est bien là, comme le souligne la citation mise en exergue par une étudiante (11, p.21):

«Il est sans doute difficile de cerner la notion de chefs-d'œuvre. Mais il est encore plus difficile d'enseigner les chefs-d'œuvre. C'est-à-dire de faire goûter, apprécier, aimer ce que j'aime et admire, ce que je place au sommet de mon panthéon personnel, à toi qui n'es plus moi et qui ne regardes pas ce que je vois de la même place, à toi dont la sensibilité ne vibre déjà plus à ce qui me faisait vibrer $\aleph^{14}$. pédagogiques de l'étude de l'image, le plus souvent pensés à l'oral. Parler sur une image «n'apparait pas pour l'élève comme un travail aussi important que comprendre un texte » explique une étudiante $(2$, p.8) : cette analyse traduit une certaine ambiguïté car, si cette donnée peut participer de la sécurisation de l'élève, elle peut également amener à l'esquive de l'apprentissage. Cette difficulté, le fait que les cours centrés sur les images en situation d'oral apparaissent comme moins sérieux aux élèves, rendant la gestion de la parole plus difficile, est pointée par plusieurs étudiants, enseignants en formation, qui ont parfois du mal à gérer ces situations.

34 À ce propos, une étudiante propose une réflexion très pertinente sur les méthodes d'approche de l'objet en histoire des arts, qui participe quelque peu d'une réflexion transdidactique. Ayant choisi pour son mémoire d'aborder la discipline du français par la trace écrite, elle signale qu'une séance observée (analyse de Guernica, en préparation à l'épreuve d'histoire des arts du brevet national des collèges), basée sur la participation orale des élèves et le travail collectif, pourrait être rendue plus efficace par un travail individuel à même le tableau, facilement réalisable à partir d'une saisie de diapositive du 
diaporama utilisé et reproduite pour les élèves, permettant par là une mise en mémoire : «Il eut été intéressant de faire un diaporama, et de plaquer en rouge les lignes de fuite. Cette méthode aurait permis aux élèves de visualiser le travail de l'artiste: de se le remémorer rapidement, rien qu'en regardant leur polycopié. On insiste toujours sur le travail à même le texte, pourquoi ne pas l'appliquer au tableau? » (10, p.12).

Au-delà de ces difficultés, de quelles pratiques didactiques observées ou construites par les étudiants témoignent de façon positive les mémoires?

\section{2. Approche motivationnelle des œuvres : les corpus de début de séance}

Il s'agit de la pratique la mieux attestée dans notre corpus, peut-être proche des rituels de création d'un horizon d'attente par un corpus de premières de couverture, pratique prenant en compte tant les questions de réception littéraire que les données de la psychologie cognitive. Ainsi, l'entrée dans l'étude de la Genèse par un document iconographique rapprochant des tableaux (Adam et Eve (1526) ou Adam et Eve au Paradis de Luca Cranach l'Ancien), la reproduction de la fresque de la chapelle Sixtine : La création d'Adam de Michel Ange juxtaposant (1510), des affiches de publicité en intertextualité avec ces domaines, une image tiré du dessin animé des Simpson, la bande-annonce de la série télévisée Desperate Housewives proposée par une étudiante. Il s'agit bien d'un choix en accord avec l'objet de l'apprentissage et l'étudiante note ses effets sur la motivation perceptibles par les nombreuses réactions suscitées.

\section{V.3. Le discours incitatif sur l'œuvre et la prise en compte de la réception personnelle d'une œuvre picturale}

37 La réception personnelle, l'émotion esthétique sont plus rarement prises en compte en tant que telles dans les dispositifs autrement que par les questionnaires ou les grilles de lecture de référence empruntée à l'instruction officielle sur l'histoire des arts. Une étudiante observe cependant les effets productifs de l'intégration de cette réception, dans un dispositif intégrant les TICE, dans le cadre de la création d'un musée de la Renaissance (exploration des enjeux esthétiques, artistiques, philosophiques et historiques de l'humanisme). Ce sont les élèves qui sélectionnent les œuvres à mettre dans ce musée : ils doivent les présenter à la classe, par groupes, à l'oral, avec des pistes d'élargissement destinées à croiser les approches, en suscitant l'envie d'étudier ces œuvres. La présentation des œuvres doit s'ancrer tout d'abord dans les impressions personnelles des élèves, qui doivent en faire état avant une étude plus précise. Ces impressions personnelles sont objets de débat avec la classe : « [...] grâce aux pistes d'élargissement, et à la consigne donnée de livrer des impressions personnelle quant à l'œuvre, les visions différaient nécessairement, ou s'enrichissaient les unes les autres. » (13 p.25). Dans une deuxième phase, à l'écrit, chaque élève doit revenir sur une des œuvres étudiée dans son groupe puis sur une autre œuvre présentée par un autre groupe. "Dans cet exercice, l'élève revient sur ce qu'il a fait dans un premier temps pour le corriger à la lumière de ce qui a été dit en classe.» (13, p.25) Dispositif qui met en avant la réception propre de l'élève et qui est susceptible de faire prendre conscience de la dimension esthétique de l'œuvre. Le discours de référence, ici, est bien plutôt le discours incitatif préconisé par Dumortier (2011) et qu'il serait intéressant de voir mis en parallèle avec un discours de 
cette nature produit par la réception d'œuvres littéraires, dans une démarche inter ou transdisciplinaire.

\section{V.4. L'approche hyperartistique ou intersémiotique des arts} poème Liberté d'Éluard (1942) et son illustration par Fernand Leger (1953) (5). Elle trouve là un point d'appui dans cette référence croisée à une double pratique d'expert, de la poésie et de la peinture, pour faire lire et écrire de la poésie et de l'art visuel. Pour modifier les représentations négatives des élèves sur la poésie, elle choisit de s'appuyer sur ce modèle pour faire écrire un poème et le présenter sur une affiche, dispositif qui intervient à la fin d'une séquence consacrée en 3e à l'étude de la poésie engagée, en lien avec l'histoire des arts. Même si l'analyse de sémiologie comparée mériterait d'être ici plus poussée, et donc outillée par la formation en amont, le choix effectué s'appuie sur une pratique artistique de référence qui ouvre des perspectives intéressantes pour penser les tâches, les productions scolaires d'une éducation esthétique (Chabanne et Dufays, 2011).

41 On peut le mettre en relation avec un travail sur Ubu roi, observé dans une classe par une étudiante (approche intersémiotique $\mathrm{BD} /$ texte de théâtre et référée au travail du metteur en scène). Un sujet est donné en préparation personnelle, faisant endosser aux élèves le rôle d'un metteur en scène, préparant sa mise en scène (notes et croquis sur le personnage d'Ubu, physique, costumes, gestuelle, voix, énonciation, déplacements etc.). Cette préparation est suivie de l'étude de la BD Ubu roi (Luc Duthil et A. Petit, 2007). L'étude précise de la BD permet de réfléchir à la représentation, aux choix scénographiques et dramaturgiques et renvoie à la lecture du texte à la lumière de cette interprétation. Il y a ici un vrai travail interdisciplinaire (ou transdisciplinaire ici?) d'esthétique comparée et une ouverture à la représentation. L'élève, quant à elle, a opté pour la confrontation de deux mises en scène de Phèdre, pour confronter les choix esthétiques des metteurs en scène, confrontation qui sert aussi à déconstruire des 
représentations : l'étudiante signale que des élèves ont ainsi compris que Racine n'était pas un homme de l'antiquité.

\section{V.5. Histoire de l'art et approche hyperartistique ou intersémiotique des arts}

Un mémoire illustre de façon tout-à-fait remarquable une interdisciplinarité assez bien maitrisée, voire une confrontation des arts à orientation épistémologique et culturelle, bien ancrée dans la logique pédagogique de la séquence, au service de la construction d'une culture commune. Il s'agit d'une séquence sur l'odyssée (2), spécifiquement sur l'épisode des Sirènes. Notons que cette étudiante a suivi un enseignement d'arts plastiques au lycée. Elle utilise avec à propos différentes catégories de supports et de productions artistiques (photos de représentations murales, de poteries (vase grec du Ve siècle), photos de sculptures : de l'antiquité (2), d'autres époques: Homère sculpté par Philippe-Laurent Roland (1812), tableaux: Ulysse et les sirènes de L.-A. Adolphe Belly (1867), Ulysse et les sirènes de John William Waterhouse (1891), Ulysse et les sirènes de Herbert Draper (1909),la bande-dessinée L'Odyssée de Christophe Lemoine et M. L. Imbiriba (2009, ed. Glenat), le film Pirate des Caraibes, la fontaine de jouvence de R. Marshall (2011). Elle analyse leur place et/ou rôle dans la séquence : mobilisation de l'attention, facilitateur de représentations (connaissances encyclopédiques), outil de compréhension et de différenciation, source de mémorisation : à ce sujet elle note que c'est finalement Homère sculpté par Philippe-Laurent Roland (1812), utilisé avec d'autres documents pour faire comprendre aux élèves le rôle de l'aède, qui est finalement resté en mémoire du cours (questionnaire final), œuvre qui a par ailleurs le plus marqué les élèves $(4$, p.12). Sa démarche atteste d'une réflexion orientée vers une épistémologie comparée entre la lecture et la réception des tableaux, même si celle-ci mériterait d'être davantage nourrie et étayée : «[...] l'image ne peut-elle pas dénaturer le texte en proposant d'emblée une interprétation (...) de ce qui est écrit. Lire, n'est-ce pas aussi et surtout laisser place à l'imagination ?... (...) l'image n'est au fond qu'une interprétation dans un champ de possibles beaucoup plus vaste qu'offre la matière textuelle. » (4, p.9-10). Bien sûr on voir surgir ici le préjugé selon lequel l'image, monosémique, exhiberait son interprétation qui ne serait donc pas à construire à l'inverse de la place laissée à l'imagination du sujetlecteur. On mesure donc ici les lacunes de la formation dans ce domaine. Toutefois la réflexion de l'étudiante s'oriente vers une épistémologie comparée rarement explicitement attestée dans notre corpus et dont la formation devrait s'emparer.

Le dispositif pour travailler sur la légende des sirènes est conçu de la façon suivante: choix de l'épisode qui tient compte de l'imaginaire des élèves déjà là (épisode fréquemment associé à la connaissance qu'ont les élèves de l'Odyssée); entrée par la lecture de l'extrait, chant XII, pour créer un effet de surprise par rapport aux représentations construites (sirènes à corps d'oiseaux) (déconstruction de la représentation : « Madame, il y a une erreur dans le texte !» s'exclame un élève 4, p.14 ); évolution du mythe et de la représentation des sirènes, incluant le rôle des pères de l'église dans sa pérennité et son évolution, retracé oralement par l'enseignante ( «J'ai remarqué combien raconter ce type d'évolution littéraire et artistique intéressait et captait l'attention des élèves qui, piqués par la curiosité, ont été nombreux à réagir. » (4 p.18); observation de documents iconographiques divers: photos de vases antiques pour étudier cette représentation avec retour au texte (ce qui amène à poser des questions de 
traduction et d'interprétation des textes grecs sur la dualité/ pluralité), étude comparée de tableaux de la fin du XIXe et du XXe, étude d'extraits du film de Pirates des Caraibes, la fontaine de Jouvence (image mobile et bande audio) et confrontation aux représentations étudiées précédemment.

«Le fait qu'il s'agissait d'un film a permis d'étudier l'évolution de la représentation des sirènes au sein même de la séquence [filmique]. En effet, les créatures passaient en quelques minutes d'une apparence positive, séduisante et onirique à une apparence négative, effrayante et surnaturelle (...). Par ailleurs étudier cette œuvre contemporaine a permis de faire prendre conscience aux élèves des liens entre époque et création. En effet, le caractère mutant des Sirènes apparait ici original en ce qu'il rompt avec l'image traditionnelle de la sirène. (...). L'étude du second extrait [du film] a quant à lui généré un ensemble de remarques établissant un pont entre l'étude des trois œuvres dans le cadre de l'histoire des arts et le film. En effet, un passage très court montrait les marins faisant tomber le coffre dans lequel ils transportaient une sirène. L'eau se déverse, et la créature, alors mi-femme, mipoisson, voit sa queue de poisson se transformer en jambes. (...) «Madame, c'est comme dans le tableau qu'on a vu (...) Cette remarque a été l'occasion de souligner les différences de traitement du sujet qu'implique le choix de telle ou telle forme d'art » (2, p.24).

L'évaluation finale du travail sur cet épisode a proposé l'analyse de trois premières de couverture d'éditions scolaires de l'Odyssée récentes représentant cet épisode. Un choix préférentiel justifié (jugement de goût, Dumortier, 2011) était également demandé, justifié par les élèves selon les critères de représentation naturaliste, de proximité avec le texte, du choix d'une action pour l'image.

Donc au total, une démarche très complète (au demeurant unique dans notre corpus) : approches des tâches et discours de l'amateur éclairé des arts et de la culture, de l'exégète et de l'herméneute, du sémiologue et de l'historien de l'art, du critique de cinéma, du sémiologue (sémiologie comparée des différents arts ou intersémiotique) etc.

\section{Conclusion}

Au terme de cette étude, et de ce qui, de fait, est déjà à l'œuvre, il nous semble que la formation des futurs enseignants de français ne saurait faire l'impasse sur une approche interdisciplinaire et transdisciplinaire de la culture humaniste. Ceci, on le voit bien à l'analyse de ce corpus, pour des raisons culturelles, didactiques et pédagogiques. L'approche de la réception des textes littéraires, notamment l'approche esthétique, trop souvent encore seulement intellectualisée, aurait tout à y gagner : l'émotion esthétique pourrait y retrouver sa place, sans dériver vers une culture de l'émotion où le paradigme de l'interprétation n'aurait plus sa place:
«[...] l'interprétation (littéraire) contribue centralement à ce qu'il y a de plus actif dans une culture: le soin cultivateur d'un héritage commun, approprié de façon à faire fructifier la construction collective de l'avenir. Ainsi s'établit une culture diachronique de l'interprétation, qui tout à la fois réunit et distend les générations qui se sont succédé dans l'élaboration cumulative d'un patrimoine intellectuel commun. » (Citton, 2011: 89).

Sans négliger les entrées pédagogiques et sans perdre de vue les objets proprement disciplinaire du français, la réflexion de nature épistémologique sur les différents objets artistiques devrait être accentuée dans la formation.

Dans l'actualité de la nouvelle refonte, en France, du master et du concours du second degré pour les futurs enseignants de français, on peut ainsi s'interroger sur les choix de 
spécialisation fait dès la première année pour l'oral du concours (latin ou cinéma ou théâtre ou français langue étrangère ou littérature et langue française), là où des éléments communs de formation pluri-artistique et une ouverture à l'histoire de l'art pour tous paraîtrait plus pertinente, à ce stade de la formation. Sans doute conviendra-til alors de penser ces enseignements en amont, dès l'année de licence, si cela se peut. Sinon le risque est grand d'un enseignement $d u$ " français " " ouvert à tous vents ", ne gagnant ni sur l'analyse de l'image, ni sur la lecture des textes et parmi eux, des textes littéraires et manquant, par là, la construction d'une nouvelle culture humaniste.

\section{BIBLIOGRAPHIE}

Ahr, S., (2012). Les classiques en bandes dessinées, sacrilège ou tremplin ? in De Peretti, I. et Ferrier, B. (dir.), Enseigner les « classiques » aujourd'hui, approches critiques et didactiques, 197-208.

Astolfi, J.-P., (2008). La saveur des savoirs. Issy-les-Moulineaux : ESF Éditeur.

Ardouin, I., (1995). Du dessin aux arts plastiques in Develay, M. dir. Savoirs scolaires et didactiques des disciplines, une encyclopédie pour aujourd'hui, Paris, ESF éditeur, 33-52.

Barthes, R., (1957). Mythologies, Paris, Le Seuil.

Barthes, R., (1964). « Rhétorique de l'image », Communication 4.

Barthes, R., (1975). « En sortant du cinéma », Communication 23.

Bautier, É. \& Rochex, J.-Y. (Éd.), (1998). L'expérience scolaire des nouveaux lycéens : démocratisation ou massification? Paris : Armand Colin.

Beaudrap, A.-R. (de) (2004). Images de la littérature et de son enseignement. Nantes : CRDP pays de la Loire.

Chabanne, J.-C. \& Dufays, J.-L., (2011). Parler et écrire sur les œuvres littéraires : contours et enjeux d'une problématique. in J.-C. Chabanne \& , J.-L. Dufays (2011) (dir.). Parler et écrire sur les œuvres : une approche interdidactique des enseignements artistiques et culturels, Repères 43 , Lyon, ENS, 7-29.

Chabanne, J.-C., Parayre, M. \& Villagordo, E. (2011). Premiers pas dans la parole sur l'œuvre : observer, interpréter et guider les conduites langagières comme compétence professionnelle, in Chabanne, J.-C. \& Dufays, J.-L. (dir.). Parler et écrire sur les œuvres : une approche interdidactique des enseignements artistiques et culturels, Repères 43, Lyon, ENS, 77-97.

Citton, Y., (2010). L'avenir des humanités. Économie de la connaissance ou cultures de l'interprétation? Paris, Éditions La découverte.

Develay, M. dir., (1995). Savoirs scolaires et didactiques des disciplines, une encyclopédie pour aujourd'hui, Paris, ESF éditeur.

Dumortier, J.-L., Dispy, M. et Van Beveren, J., (2011). Pour le discours incitatif, in Chabanne, J.-C. \& Dufays, J.-L. (dir.). Parler et écrire sur les œuvres : une approche interdidactique des enseignements artistiques et culturels, Repères 43, Lyon, ENS, 31-52. 
Fourtanier, M.-J., (2013). Lire et faire lire des œuvres littéraires adaptées en BD : ravauder le patrimoine ou l'inventer? in Denizot, N. et Ahr, S. (dir.), Dyptique 26, Namur, PUN, 33-50.

Genette, G., (1982). Palimpsestes. Paris, Éditions du Seuil.

Louichon, B., (2012). Définir la littérature patrimoniale, in De Peretti, I. et Ferrier, B. (éd.), Enseigner les « classiques » aujourd'hui. Approches critiques et didactiques, 37-49). Bruxelles : Peter Lang.

Musset, M., Dossier d'actualité $\mathrm{n}^{\circ} 33$, février 2008. Service de veille scientifique et technologique, www.inrp.fr/vst, consulté le08/09/2008.

Metz, C., (1970). « Images et Pédagogie » et « Au-delà de l'analogie, l'image », Communication 15.

Kalhart, P., (2002). Autour des images. Paris, Bourrelier.

Reuter, Y., (1995). Vers une synthèse : réflexions et propositions, in Chiss, J.-L, David, J. et Reuter, Y. (dir.), Didactique du français, État d'une discipline. Paris : Nathan pédagogie.

Veck, B., (1994). La culture littéraire au lycée : des humanités aux méthodes ? Paris : INRP.

\section{NOTES}

1. BOEN n ${ }^{\circ} 29$ du 20 juillet 2006 : Socle commun de connaissances et de compétences (lui-même en lien avec les orientations de l'Europe en matière d'éducation), BOEN n 32 du 28 août 2008 : Organisation de l'histoire des arts, BOEN spécial n 6 du 28 août 2008 Programmes du collège.

2. L'évaluation de l'histoire des arts (une épreuve orale) est prise en compte pour l'obtention du diplôme national du brevet des collèges (DNB), depuis la session de 2012 selon des modalités définies par la circulaire $\mathrm{n}^{\circ} 2011$ - 189 du 3-11-2011, parue au BOEN nº 41 du 10 novembre 2011 .

3. (telle qu'elle est définie dans le pilier 5 de l'instruction du Socle commun de connaissances et de compétences),

4. Nous nous référons ici également aux définitions que J.-P. Astolfi donne de ces notions dans La saveur des savoirs, pp.109-115 : la pluridisciplinarité, qui juxtapose les approches, se trouve du côté de la motivation ; l'interdisciplinarité articule dans la conscience claire de la spécificité de leurs objets et de leurs méthodes des disciplines différentes les disciplines convergeant de façon fonctionnelle pour l'étude d'un objet précis; la transdisciplinarité ou épistémologie comparée examine les transferts conceptuels et les enrichissements mutuels des différentes disciplines, elle favorise comparaisons et confrontations. Dans la culture humaniste, de fait, suivant en cela les mémoires des étudiants, nous examinerons plutôt les liens entre littérature, arts visuels et histoire de l'art ou des arts.

5. BOEN spécial $n^{\circ} 6 \mathrm{du} 28$ août 2008 : Programmes du collège.

6. BOEN $n^{\circ} 29$ du 20 juillet 2006 : Socle commun de connaissances et de compétences.

7. BOEN no 32 du 28 août 2008 : Organisation de l'histoire des arts.

8. Ces enseignements ont disparu, de fait, avec les nouvelles maquettes de 2013/2014, adaptées aux nouveaux objectifs du Capes de lettres modernes.

9. Les chiffres 1 à 18 cités entre parenthèses renvoient bien sûr à ces 18 mémoires.

10. Titres des mémoires $4:$ «L'image dans l'enseignement du français »; 2 «L'image, auxiliaire de la discipline du français ? "; 10 «L'image comme déclencheur de l'écriture »;11 « Motiver par l'histoire des arts".

11. Titres des autres mémoires : 7 « La trace écrite »; 6 « La variété des supports »; «La place des Technologies de l'information et de la communication en cours de français : une révolution technologique?»; 13 «Enseigner le processus d'auto-évaluation pour préparer l'EAF : de la clarification des enjeux de l'évaluation à l'autonomisation des apprenants »; 17 «La 
compréhension et le goût de la lecture au collège »; 8 « Quelle représentation les élèves ont-ils de l'écrivain ?»; 5 « Enseigner la poésie engagée en classe de troisième ou comment modifier les représentations des élèves grâce à l'écriture »; 9 « Ce qui motive ou rebute l'élève à la lecture des pièces de théâtre ».

12. Pour reprendre la typologie de L'organisation de l'histoire des arts (2008).

13. Sylvie Anahory, "L'image pour comprendre les textes", dans Les arts, quelle histoire!, dossier coordonné par B. Duvin Parmentier et C. Vallin, Paris, Cahiers pédagogiques, $\mathrm{n}^{\circ} 492$, novembre 2011, p. 30-31.

14. Eluard, P. CEuvres complètes, "Poèmes politiques ", «Portrait ", Paris, Gallimard, «La Pléiade ", 1996, 2 vol., p. 212.

15. Nous empruntons les catégories du faire, voir, comprendre, caractéristique du paradigme actuels des arts visuels à I. Ardouin, op. cit. , 1995.

\section{RÉSUMÉS}

Il semble bien difficile aujourd'hui de voir clair dans toute tentative de bilan de la formation dispensée dans les masters MEEF du premier ou du second degré, en ce qui concerne la formation à l'enseignement de la littérature. Tant ce qui peut être potentiellement ferment positif se voit immédiatement dévalué par les conditions catastrophiques de la mise en œuvre de la mastérisation et tant le recul manque pour apprécier l'impact de tel ou tel nouveau contenu d'enseignement. On s'attachera pourtant ici, pour poser quelques jalons et à titre exploratoire, à suivre les effets, dans les mémoires professionnels (premier et second degrés), de l'enseignement dispensé dans les séminaires de recherche en didactique de la littérature durant la première année de la réforme.

It seems very difficult today to see clearly in any attempt of balance sheet of the training dispensed in the Master's degrees MEEF of the first one or the second degree, as regards the training in the education of the literature. So much what can be potentially positive ferment sees itself immediately devalued by the catastrophic conditions of the implementation of the mastering and so much the backward movement is lacking to appreciate the impact of such or such new contents of education. We shall nevertheless attempt here, to put some milestones and in exploratory title, to follow the effects, in the professional reports (first and second degrees, the education exempted in the seminaries of search in didactics of the literature during the first year from the reform.

\section{INDEX}

Mots-clés : didactique de la littérature, formation à l'enseignement de la littérature, mémoires professionnels

Keywords : didactics of the literature, professional reports, raining in the education of the literature 


\section{AUTEUR}

\section{ISABELLE DE PERETTI}

ESPE Lille Nord de France, Artois EA 4028 